\title{
ASYMPTOTIC BEHAVIOR OF SOLUTIONS OF LINEAR STOCHASTIC DIFFERENTIAL SYSTEMS $\left({ }^{1}\right)$
}

BY

\author{
AVNER FRIEDMAN AND MARK A. PINSKY
}

\begin{abstract}
Following $\mathrm{Kasminski,} \mathrm{we} \mathrm{investigate} \mathrm{asymptotic} \mathrm{behavior} \mathrm{of}$ solutions of linear time-independent Itô equations. We first give a sufficient condition for asymptotic stability of the zero solution. Then in dimension 2 we determine conditions for spiraling at a linear rate. Finally we give applications to the Cauchy problem for the associated parabolic equation by the use of a tauberian theorem.
\end{abstract}

Introduction. Consider a system of linear, constant coefficients differential equations

$$
\frac{d x_{i}}{d t}=\sum_{j=1}^{l} b_{i}^{j} x_{j} \quad(1 \leq i \leq l) .
$$

It is well known that the solutions of $(0.1)$ are asymptotically stable if and only if the eigenvalues of $\left(b_{i}^{j}\right)$ have negative real part. In case $l=2$ the angular behavior can be determined, leading to the familiar cases of focal points, nodal points and saddle points.

The corresponding problem for a system of Itô stochastic differential equations

$$
d x_{i}=\sum_{j=1}^{l} \sum_{r=1}^{n} \sigma_{i r}^{j} x_{j} d w^{r}+\sum_{j=1}^{l} b_{i}^{j} x_{j} d t \quad(1 \leq i \leq l)
$$

was first studied by Kasminski [5]. He decomposed the stochastic process into its radial part $r(t)=|x(t)|$ and its "angular" part $\lambda(t)=x(t) /|x(t)|$. Assuming a strong ergodicity condition for $\{\lambda(t), t \geq 0\}$, he gave a necessary and sufficient condition that $r(t) \rightarrow 0$ a.s. when $t \rightarrow \infty$. He did not examine, however, the be havior of $\{\lambda(t), t>0\}$. The first study of the angular behavior in the case $l=2$

Received by the editors March 13, 1972.

AMS (MOS) subject classifications (1970). Primary 60H10, 60J60; Secondary 34D05, 34A30, 34C05.

Key words and phrases. Stochastic differential equation, diffusion process, asymptotic stability, spiraling solutions.

(1) This work was partially supported by National Science Foundation grants GP-28484 and GP-9437. 
was carried out by Kozin and Prodromou [6] who proved the existence of spirals for certain examples.

The main contribution of the present paper is to the study of the angular behavior of $\{x(t), t \geq 0\}$ in the general case $l=2$. The homogeneous form of $(0.2)$ implies (see $\$ 2)$ that $\{\lambda(t)=(\cos \phi(t), \sin \phi(t)), t \geq 0\}$ is a diffusion process which can be determined by a single Itô equation on $(-\infty, \infty)$ :

$$
d \phi=\tilde{\sigma}(\phi) d w+\widetilde{b}(\phi) d t .
$$

In case $\tilde{\sigma}(\phi)$ is never zero, the asymptotic behavior depends upon

$$
\widetilde{\Lambda}=\int_{0}^{2 \pi} \frac{\tilde{b}(\phi)}{\tilde{\sigma}^{2}(\phi)} d \phi .
$$

We prove (Theorem 3.1) that if $\tilde{\Lambda}>0(\tilde{\Lambda}<0)$ then a.s.

(0.5) $\quad \lim _{t \rightarrow \infty} \frac{\phi(t)}{t}$ exists and is a positive (negative) constant.

In case $\tilde{\sigma}(\phi)=0$ for some points $\phi$, we need to examine the sign of $\tilde{b}(\phi)$ at these points. If, for instance, $\tilde{b}(\phi)>0(\tilde{b}(\phi)<0)$ at all the points where $\widetilde{\sigma}(\phi)=0$, then (Theorem 3.3) the assertion (0.5) again holds. Although this result may be derived by employing the general classification of one-dimensional diffusion, we give a new method of proof based on constructing certain "comparison functions" for the differential generator of the angular process.

Finally, we compare the behavior of the solutions of $(0.2)(l=2)$ with the behavior in the deterministic case, achieving the surprising result (Theorem 5.2) that if $\widetilde{\sigma}(\phi)$ does vanish at some points then, whenever the solutions of $(0.1)$ spiral, the same is true of the solutions of (0.2).

In the final section we apply our results to the Cauchy problem

$$
\partial u / \partial t=L u \quad\left(t>0, x \in R^{l}\right), \quad u(0, x)=f(x) \quad\left(x \in R^{l}\right),
$$

where $L$ is the differential generat or of the diffusion process of $(0.2)$ and $l=2$. We prove that whenever the solutions of $(0.2)$ spiral, $\lim _{t \rightarrow \infty} u(t, x)$ exists provided $f(x)=g(\phi)(x=(r \cos \phi, r \sin \phi))$. We also obtain results on the domain of dependence of the solution upon the initial data.

The asymptotic behavior of solutions of nonlinear stochastic differential equations was studied in Freidlin [1] and in Friedman [3]. The limit theorems obtained in these papers are of entirely different nature than those obtained in the present work.

1. Properties of the radial motion. Consider a system of linear stochastic differential equations 


$$
\begin{aligned}
d x_{i} & =\sigma_{i r}^{j} x_{j} d w^{r}+b_{i}^{j} x_{j} d t \quad(1 \leq i \leq l), \\
x_{i}(0) & =x_{i}^{0}
\end{aligned}
$$

where $\left(w^{1}(t), \cdots, w^{n}(t)\right)$ are independent Brownian motions and $\sigma_{i r}^{j}, b_{i}^{j}$ are constants, $1 \leq i, j \leq l, 1 \leq r \leq n$; we use the summation convention throughout. It is always tacitly assumed that $x(0) \neq 0$.

Kasminski [5] has observed that the solution of (1.1) can be written as $x_{i}(t)=r(t) \lambda_{i}(t)$ where $\left(\lambda_{1}(t), \ldots, \lambda_{l}(t)\right)$ is a Markov process on the sphere $|\lambda|=1$ and $r(t)=|x(t)|$ is an explicit functional of $\{\lambda(t), t \geq 0\}$. In the case where the $\lambda$-process is ergodic, Kasminski presented a necessary and sufficient condition that $r(t) \rightarrow 0$ when $t \rightarrow \infty$. That condition, however, is of very limited use since it requires an explicit knowledge of the invariant measure of the $\lambda$-process. We shall derive in this section a cruder but more easily verified stability condition which does not depend on the ergodicity of the $\lambda$-process.

The differential generator of the $x(t)$ process is

$$
L u \equiv\langle B x, \operatorname{grad} u\rangle+\frac{1}{2} \sum_{i, j=1}^{l} a_{i j}(x) \frac{\partial^{2} u}{\partial x_{i} \partial x_{j}},
$$

where

$$
a_{i j}(x)=\sum_{k, s=1}^{l} \sum_{r=1}^{n} \sigma_{i r}^{k} \sigma_{j r}^{s} x_{k} x_{s}, \quad B=\left\|b_{i}^{j}\right\| \cdot
$$

If $u=\phi(r)$ where $r=|x|$, then

$$
L \phi(r)=r \phi^{\prime}(r) Q(\lambda)+1 / 2\left[r^{2} \phi^{\prime \prime}(r)+r \phi^{\prime}(r)\right]\langle a(\lambda) \lambda, \lambda\rangle,
$$

where $\lambda_{i}=x_{i} /|x|$ and

$$
Q(\lambda)=\langle B \lambda, \lambda\rangle+1 / 2 \operatorname{tr} a(\lambda)-\langle a(\lambda) \lambda, \lambda\rangle \cdot
$$

Let us show as a preliminary step that, in general, the $x(t)$ process does not hit the origin.

Theorem 1.1. $P\{\exists t>0$ for which $x(t)=0\}=0$.

Proof. Let $\phi(r)=r^{-\epsilon}$ where $\epsilon$ is a fixed number in the interval $(0,1)$. Using (1.4) we get

if

$$
L \phi=\epsilon \phi[-Q(\lambda)+(\epsilon / 2)\langle a(\lambda) \lambda, \lambda\rangle]<\mu \phi
$$

$$
\mu>\sup _{|\lambda|=1}\{|Q(\lambda)|+1 / 2\langle a(\lambda) \lambda, \lambda\rangle\} .
$$

By Itô's formula, if $f(x)$ is in $C^{2}\left(R^{n}\right)$ then 


$$
\begin{aligned}
& e^{-\mu t^{*}} f\left(x\left(t^{*}\right)\right) \\
& \quad=f\left(x_{0}\right)+\sum_{i, j, r} \int_{0}^{t^{*}} e^{-\mu s} f_{x_{i}}(x(s)) \sigma_{i r}^{j} x_{j}(s) d w^{r}(s)+\int_{0}^{t^{*}}(L f-\mu f) e^{-\mu s} d s
\end{aligned}
$$

where $t^{*}$ is any bounded Markov time.

Now let $\left\{r_{n}\right\}$ be a decreasing sequence of radii tending to zero, and let $f_{n}(x)$ be a $C^{2}\left(R^{l}\right)$ function satisfying $f_{n}(x)=r^{-\epsilon}$ for $r>r_{n}$. Set $x_{0}=x(0)$. If $\left|x_{0}\right|>r_{n}$ and $T_{n}=\inf \left\{t ;|x(t)|=r_{n}\right\}$ then, by (1.5) with $f=f_{n}$ and $t^{*}=t \wedge T_{n}$,

$$
E\left\{e^{-\mu\left(T_{n} \wedge t\right)} f_{n}\left(x\left(t \wedge T_{n}\right)\right)\right\}=f\left(x_{0}\right)+E \int_{0}^{t \wedge T_{n}}(L f-\mu f) e^{-\mu s} d s \leq f\left(x_{0}\right) .
$$

Let $B$ be the event $\left\{\omega ; \inf _{t>0}|x(t)|=0\right\}$. Letting $t \rightarrow \infty$ in the last inequality and noting that $f\left(x\left(t \wedge T_{n}\right)\right)=r_{n}^{-\epsilon}$ if $t>T_{n}$, it follows, by the monotone convergence theorem, that

$$
E\left\{e^{-\mu T} \chi_{B}\right\} / r_{n}^{\epsilon} \leq 1 /\left|x_{0}\right|^{\epsilon}
$$

Taking $n \rightarrow \infty$, we obtain

$$
E\left\{e^{-\mu T} \chi_{B}\right\}=0 \text { where } T=\lim _{n \rightarrow \infty} T_{n} .
$$

Hence $T=\infty$ a.s. on the set $B$. Thus, for almost all $\omega \in B, x(t)$ does not intersect 0 at a finite time. Since for any $\omega \notin B, x(t) \neq 0$ for all $t>0$, the proof of the theorem is complete.

The next theorem establishes a simple sufficient condition for stability.

Theorem 1.2. If $\sup _{|\lambda|=1} Q(\lambda)=-a<0$ then, for some constant $C$,

$$
\begin{gathered}
E\{\log r(t)\} \leq C-\alpha t, \\
P\left\{\varlimsup_{t \rightarrow \infty} \frac{\log r(t)}{t} \leq-\alpha\right\}=1 .
\end{gathered}
$$

Similarly, if inf $|\lambda|=1$ Q $Q(\lambda)=\beta>0$ then, for some constant $C$,

$$
\begin{gathered}
E\{\log r(t)\} \geq-C+\beta t, \\
P\left\{\frac{\lim }{t \rightarrow \infty} \frac{\log r(t)}{t} \geq \beta\right\}=1 .
\end{gathered}
$$

Proof. By Theorem 1.1, $\log r(t)$ is a well-defined random variable for any $t>0$. From Itô's formula and (1.4)

$$
\log r(t)=\log r(0)+\int_{0}^{t} \frac{x_{i}}{r^{2}} \sigma_{i r}^{j} x_{j} d w^{r}(s)+\int_{0}^{t} Q(\lambda(s)) d s .
$$

Since the Itô integral has mean zero, whereas the Lebesgue integral is $\leq-a t$, the inequality (1.6) follows. To prove (1.7), we use the following lemma. 
Lemma 1.3. Let $\sigma(t)$ be a nonanticipative functional of $\{w(t), t \geq 0\}$, $|\sigma(t)| \leq K$. Then, a.s.,

$$
\frac{1}{t} \int_{0}^{t} \sigma(s) d w(s) \rightarrow 0 \text { when } t \rightarrow \infty
$$

From this lemma it follows that a.s.

$$
\frac{1}{t} \int_{0}^{t} \frac{x_{i}^{x} j}{r^{2}} \sigma_{i r}^{j} d w^{r}(s) \rightarrow 0 \text { if } t \rightarrow \infty .
$$

Since also $\int_{0}^{t} Q(\lambda(s)) d s \leq-\alpha t$, the assertion (1.7) follows. The proof of (1.8), (1.9) is similar.

Proof of Lemma 1.3. Let $M>K$ and define

$$
T_{t}=\int_{0}^{t}(\sigma(s)+M)^{2} d s, \quad a(t)=\int_{0}^{T_{t}^{-1}}(\sigma(s)+M) d w(s) .
$$

(Since $\sigma+M>0, a(t)$ is well defined.) By [4, p. 32] or [7, p. 29], $\{a(t), t \geq 0\}$ is a Brownian motion. Consequently, $a(t) / t \rightarrow 0$, or $a\left(T_{t}\right) / T_{t} \rightarrow 0$, when $t \rightarrow \infty$. Noting that $T_{t} \leq(K+M)^{2} t$, it follows that

$$
\frac{1}{t} \int_{0}^{t}(\sigma(s)+M) d w(s) \rightarrow 0 \quad \text { if } t \rightarrow \infty
$$

Since $w(t) / t \rightarrow 0$ when $t \rightarrow \infty$, (1.11) follows.

2. The case $l=2$; reduction to one equation. We now restrict ourselves to systems in the plane, i.e., $l=2$. We introduce polar coordinates $(r, \phi)$ by $x=$ $r \cos \phi, y=r \sin \phi$. From (1.1) we may formally compute the stochastic differential of $\phi$ :

$$
d \phi=\phi_{x} d x+\phi_{y} d y+1 / 2 \phi_{x x} a_{11} d t+\phi_{x y} a_{12} d t+1 / 2 \phi_{y y} a_{22} d t
$$

Noting that

we find that

$$
\begin{aligned}
& \phi_{x}=-(\sin \phi) / r, \quad \phi_{y}=(\cos \phi) / r, \quad \phi_{x x}=(2 \sin \phi \cos \phi) / r^{2}, \\
& \phi_{x y}=\left(\sin ^{2} \phi-\cos ^{2} \phi\right) / r^{2}, \quad \phi_{y y}=-(2 \sin \phi \cos \phi) / r^{2},
\end{aligned}
$$

$$
d \phi=\left\langle\sigma_{r} \lambda, \lambda^{\perp}\right\rangle d w^{r}-\left\{\left\langle a(\lambda) \lambda, \lambda^{\perp}\right\rangle-\left\langle B \lambda, \lambda^{\perp}\right\rangle\right\} d t
$$

where $\sigma_{r}=\left\|\sigma_{r j}^{i}\right\|, \lambda=(\cos \phi, \sin \phi), \lambda^{\perp}=(-\sin \phi, \cos \phi)$.

The above has the following meaning: equation (2.1), with initial condition $\phi(0)=\phi_{0}$, defines a diffusion process in the interval $-\infty<\phi<\infty$. In terms of this process and the Brownian motions $\left(w^{1}(t), \ldots, w^{n}(t)\right)$, we define a process $r(t)$ by the equation

$$
r(t)=r_{0} \exp \left\{\int_{0}^{t}\left\langle\sigma_{r} \lambda(s), \lambda(s)\right\rangle d w^{r}(s)\right\} \exp \left\{\int_{0}^{t} Q(\lambda(s)) d s\right\}
$$


where $\lambda(s)=(\cos \phi(s), \sin \phi(s))$; this is motivated by (1.10). The pair $y(t)=$ $(r(t), \phi(t))$ is a diffusion process on $(0, \infty) \times(-\infty, \infty)$. We now define $x(t)=$ $\left(x_{1}(t), x_{2}(t)\right)$ where $x_{1}(t)=r(t) \cos \phi(t), x_{2}(t)=r(t) \sin \phi(t)$. We claim

Theorem 2.1. $\{x(t), t \geq 0\}$ is a diffusion process which can be obtained as a solution of (1.1).

Proof. We have to verify the stochastic equations (1.1) for $x(t)$. We resort to the general reasoning:

Write $x_{i}(t)=g^{i}(y)$ where $y=\left(y_{1}, y_{2}\right)$ and $g$ is a global differentiable transformation $(r, \phi) \rightarrow(r \cos \phi, r \sin \phi)$. The stochastic differential of $x$ can be computed by Itô's formula (subscripts following commas denote partial derivatives)

$$
d x_{i}=g_{, k}^{i} d y_{k}+1 / 2 g_{, k l}^{i} d y_{k} d y_{l}
$$

On the other hand, by definitions (2.1), (2.2), the stochastic differentials of. $\left(y_{1}, y_{2}\right)=(r, \phi)$ were obtained in terms of the local inverse of $g: x \rightarrow f(x)=$ $\left(\left(x_{1}^{2}+x_{2}^{2}\right)^{1 / 2}, \tan ^{-1}\left(x_{2} / x_{1}\right)\right)$; thus

$$
\begin{aligned}
d y_{k} & =f_{, i}^{k} d x_{i}+1 / 2 f_{, i j}^{k} d x_{i} d x_{j} \\
& =f_{, i}^{k}(g(y))\left\{\sigma_{i r}(g(y)) d w^{r}+b_{i}(g(y)) d t\right\}+1 / 2 f_{, i j}^{k}(g(y)) \sigma_{i r}(g(y)) \sigma_{j r}(g(y)) d t .
\end{aligned}
$$

$\left(\sigma_{i r}(z)=\sigma_{i r}^{j} z_{j}, b_{i}(z)=b_{i}^{j} z_{j}\right)$ is the stochastic system defining the $y$-process. If we now substitute (2.4) into (2.3), we get (omitting the arguments of $g, f, \sigma, b$ )

$$
d x_{i}=g_{, k}^{i}\left[f_{, l}^{k} \sigma_{l r} d w^{r}+\left(1 / 2 f_{, i j}^{k} \sigma_{i r} \sigma_{j r}+f_{, i}^{k} b_{i}\right) d t\right]+1 / 2\left(g_{, k l}^{i} f_{, p}^{k} \sigma_{p r} f_{, q}^{l} \sigma_{q r}\right) d t .
$$

Since $f$ and $g$ are inverse functions (locally), it follows (by differentiating once the relation $f(g(y))=y$ ) that $f_{, i}^{k} g_{, l}^{i}=\delta_{k l}$ and (by differentiating once the relation $\left.f_{, l}^{k} g_{, k}^{i}=\delta_{i l}\right)$ that $g_{, k}^{i} f_{, l m}^{k}+f_{, l}^{k} g_{, k p}^{i} f_{, m}^{p}=0$. Using these relations in the last expression for $d x_{i}$, we arrive at $d x_{i}=\sigma_{i r} d w^{r}+b_{i} d t$. This proves the theorem, and thus completes the identification of the solution of (2.1) with the algebraic angle of the original diffusion process $x(t)$.

As a final reduction we show that with a single Brownian motion we can obtain a process equivalent, in the sense of joint distributions, to (2.1). Let

$$
\begin{aligned}
& \tilde{\sigma}(\phi)=\left(\sum_{r=1}^{n}\left\langle\sigma_{r} \lambda, \lambda^{\perp}\right\rangle^{2}\right)^{1 / 2}, \\
& \tilde{b}(\phi)=\left\langle B \lambda, \lambda^{\perp}\right\rangle-\left\langle a(\lambda) \lambda, \lambda^{\perp}\right\rangle
\end{aligned}
$$

where $a$ and $B$ are as in (2.1). The functions $\tilde{\sigma}, \tilde{b}$ are Lipschitz continuous periodic functions with period $2 \pi$. Let $\phi_{1}(t)$ be the solution of

$$
\phi(t)=\phi_{0}+\int_{0}^{t} \tilde{\sigma}(\phi(s)) d w(s)+\int_{0}^{t} \tilde{b}(\phi(s)) d s
$$


where $\{w(t), t \geq 0\}$ is a Brownian motion. An immediate calculation using Itô's formula shows that $\phi(t)$ and $\phi_{1}(t)$ have the same generator

$$
\widetilde{L}=1 / 2(\widetilde{\sigma}(\phi))^{2} d^{2} / d \phi^{2}+\widetilde{b}(\phi) d / d \phi .
$$

From the uniqueness of bounded solutions of the Cauchy problem for parabolic equations [2] it follows that $E f\left(\phi_{1}(t)\right)=E f(\phi(t))$ for any $f \in C^{2}, f$ bounded; here $\phi_{1}(0)=\phi(0)$. The Markov property now implies that the joint distribution of the processes $\phi_{1}(t)$ and $\phi(t)$ agree.

In the following two sections, we shall study equations of the form (2.7).

3. One stochastic equation. We shall consider in this section one stochastic equation

$$
d \phi=b(\phi) d t+\sigma(\phi) d w, \quad \phi(0)=\phi_{0}
$$

with uniformly Lipschitz continuous coefficients $b(\phi), \sigma(\phi)$, and with $\sigma(\phi) \geq 0$.

Theorem 3.1. Assume that $\sigma(z)>0$ for all real $z$ and that $b(z), \sigma(z)$ are periodic of period $2 \pi$. Let

$$
\Lambda=\int_{0}^{2 \pi} \frac{b(z)}{\sigma^{2}(z)} d z
$$

If $\Lambda>0$ then $T_{1}=\inf \left\{t ; \phi(t)-\phi_{0}=2 \pi\right\}$ is integrable witb $E T_{1}>0$, and

$$
P_{\phi_{0}}\left\{\lim _{t \rightarrow \infty} \frac{\phi(t)}{t}=\frac{2 \pi}{E\left(T_{1}\right)}\right\}=1 \text {. }
$$

If $\Lambda<0$ then $T_{-1}=\inf \left\{t ; \phi(t)-\phi_{0}=-2 \pi\right\}$ is integrable with $E T_{-1}>0$, and

$$
P_{\phi}\left\{\lim _{t \rightarrow \infty} \frac{\phi(t)}{t}=-\frac{2 \pi}{E\left(T_{-1}\right)}\right\}=1 \text {. }
$$

Finally, if $\Lambda=0$ then

$$
\begin{array}{r}
P_{\phi_{0}}\left\{\varlimsup_{t \rightarrow \infty} \phi(t)=\infty\right\}=1, \\
P_{\phi_{0}}\left\{\frac{\lim }{t \rightarrow \infty} \phi(t)=-\infty\right\}=1 .
\end{array}
$$

Proof. Set

$$
\Phi(z)=\exp \left\{-\int_{0}^{z} \frac{2 b(u)}{\sigma^{2}(u)} d u\right\}, \quad I_{1}=\int_{-\infty}^{0} \Phi(z) d z, \quad I_{2}=\int_{0}^{\infty} \Phi(z) d z .
$$

For $z$ positive,

$$
\int_{0}^{z} \frac{2 b(u)}{\sigma^{2}(u)} d u=2 \Lambda[z / 2 \pi]+\theta \quad\left(|\theta| \leq C^{\prime}, C^{\prime} \text { constant }\right)
$$

where $[\alpha]$ denotes the largest integer $\leq \alpha$. Therefore 


$$
\Phi(z)=\exp \{-\Lambda z / \pi+O(1)\} .
$$

The same relation holds for $z$ negative. It follows that when $\Lambda>0, I_{1}=\infty$ and $I_{2}<\infty$. By Theorem 1 of [4, p. 119] we conclude that $\lim _{t \rightarrow \infty} \phi(t)=\infty$ a.s. To prove the sharper result (3.3), we may take $\phi_{0}=0$ and write $P_{0}=P, E_{0}=E$. Define successive Markov times

$$
T_{n}=\inf \{t>0 ; \phi(t)=2 n \pi\}
$$

We claim that

$$
E\left(T_{1}\right)>\infty
$$

To prove it, denote by $\tau_{a}(a<0)$ the exit time of $\phi(t)$ from the interval $(a, 2 \pi)$. Then (see [4, p. 109])

$$
E\left(\tau_{a}\right)=-\int_{a}^{0} 2 \Phi(y) \int_{a}^{y} \frac{d z}{\sigma^{2}(z) \Phi(z)}
$$

$$
+\left\{\int_{a}^{2 \pi} 2 \Phi(y) \int_{a}^{y} \frac{d z}{\sigma^{2}(z) \Phi(z)}\right\}\left(\int_{a}^{0} \Phi(z) d z / \int_{a}^{2 \pi} \Phi(z) d z\right) .
$$

Using (3.7) one easily finds that $E\left(\tau_{a}\right) \leq C$ where $C$ is a constant independent of $a$. Since $\tau_{a} / T_{1}$ as $a \downarrow-\infty, E\left(T_{1}\right) \leq C$.

Using the strong Markov property and the periodicity of $b(z), \sigma(z)$, it follows that the random variables $\int_{T_{n-1}}^{T_{n}} b(\phi(s)) d s(n=1,2, \ldots)$ are independent and identically distributed. Since, by (3.8), also $E\left|\int_{0}^{T} 1 b(\phi(s)) d s\right|<\infty$, we can apply the strong law of large numbers to deduce that

$$
\begin{aligned}
\int_{0}^{t} b(\phi(s)) d s & =\sum_{1}^{N(t)} \int_{T_{n-1}}^{T_{n}} b(\phi(s)) d s+\int_{T_{N(t)}^{t}}^{t} b(\phi(s)) d s \\
& \sim N(t) E \int_{0}^{T_{1}} b(\phi(s)) d s
\end{aligned}
$$

where $N(t)$ is the largest $n$ such that $T_{n} \leq t$. The strong law of large numbers applies also to $\left\{T_{n}-T_{n-1}\right\}_{n=1,2}, \ldots$. It shows that $N(t) \sim c_{1} t$ as $t \rightarrow \infty\left(c_{1}\right.$ constant). Thus we have

$$
\frac{1}{t} \int_{0}^{t} b(\phi(s)) d s \rightarrow c \quad \text { if } t \rightarrow \infty \quad(c \text { constant })
$$

But by Lemma 1.3 ,

$$
\frac{1}{t} \int_{0}^{t} \sigma(\phi(s)) d w(s) \rightarrow 0 \quad \text { if } t \rightarrow \infty
$$

Consequently, $\phi(t) / t \rightarrow c$ if $t \rightarrow \infty$, a.s. In order to evaluate $c$, we sample the process at $t=T_{1}, T_{2}, \cdots$. Thus, $2 n \pi=\phi\left(T_{n}\right) \sim c T_{n} \sim c n E\left(T_{1}\right)$ where in the last relation we have used the strong law of large numbers. It follows that $2 \pi=$ $c E\left(T_{1}\right)$, and (3.3) is proved. Note that since $c$ is a real number, $E\left(T_{1}\right) \neq 0$. 
The proof of (3.4) is similar. Finally, if $\lambda=0$ then $I_{1}=I_{2}=\infty$ and (3.5), (3.6) follow from Theorem 1 of [4, p. 119].

Remark. $E\left(T_{1}\right)$ can be computed from the relations $E\left(T_{1}\right)=\lim _{a \rightarrow-\infty} E \tau_{a}$ and (3.9).

Consider next the case where $\sigma(z)$ vanishes on a sequence of points. Suppose

$$
\begin{aligned}
& \beta_{0}<\beta_{1}<\beta_{2}<\cdots<\beta_{k}<\beta_{k+1}, \\
& \sigma\left(\beta_{1}\right)=\sigma\left(\beta_{2}\right)=\cdots=\sigma\left(\beta_{k}\right)=0, \\
& \sigma(z)>0 \text { in the intervals }\left(\beta_{j}, \beta_{j+1}\right), \quad 0 \leq j \leq k .
\end{aligned}
$$

Consider first the case where

$$
b\left(\beta_{1}\right)>0, b\left(\beta_{2}\right)>0, \cdots, b\left(\beta_{k}\right)>0 .
$$

Denote by $\tau_{x}(a, b)$ the exit time of $\phi^{\prime}(t)$ from $(a, b)$, where $\phi(0)=x$. We shall prove

Theorem 3.2. Let (3.10), (3.11) bold. Then, for any $\alpha \in\left(\beta_{0}, \beta_{1}\right]$ and for any $\gamma \in\left(\dot{\beta}_{k}, \beta_{k+1}\right)$,

$$
E\left\{\tau_{x}(\alpha, \gamma)\right\}<\infty \text { if } x \in(\alpha, \gamma)
$$

Furthermore,

$$
P\left\{\phi\left(\tau_{x}\left(\beta_{1}, \gamma\right)\right)=\gamma\right\}=1 \quad \text { if } x \in\left(\beta_{1}, \beta_{k}\right) .
$$

Note, by The orem 2 of $\left[4\right.$, p. 150], that if $x=\beta_{1}$ then $\phi(t)>\beta_{1}$ a.s. for any $t>0$.

Proof. For any $\epsilon>0$ sufficiently small

$$
\begin{aligned}
& b(x) \geq b_{0}>0 \quad \text { in the intervals }\left(\beta_{i}-\epsilon, \beta_{i}+\epsilon\right), \quad 1 \leq i \leq k, \\
& \sigma(x) \geq \sigma_{0}>0 \quad \text { in the intervals }\left(\beta_{i}+\epsilon, \beta_{i+1}-\epsilon\right), \quad 0 \leq i \leq k+1,
\end{aligned}
$$

where $b_{0}$ is independent of $\epsilon$ and $\sigma_{0}$ depends on $\epsilon$. We shall construct a piecewise $C^{2}$ function $f(x)$ such that

$$
L f(x) \leq-\nu<0 \quad \text { in }(\alpha, \gamma)
$$

where $L u \equiv 1 / 2 \sigma^{2} u^{\prime \prime}+b u^{\prime} ; f$ and $\nu$ will depend only on $b_{0}, \sigma_{0}, \epsilon$ and on an upper bound on $b$.

We take $\epsilon$ such that $\beta_{0}+\epsilon<\alpha, \gamma+\epsilon<\beta_{k+1}$ and, for definiteness, suppose that $\alpha<\beta_{1}-\epsilon$. We begin by taking 


$$
f(x)= \begin{cases}(1 / k)\left[1-e^{k\left(x-\beta_{1}+\epsilon\right)}\right] & \text { in }\left(\alpha, \beta_{1}-\epsilon\right), \\ -\left(x-\beta_{1}+\epsilon\right) & \text { in }\left(\beta_{1}-\epsilon, \beta_{1}+\epsilon\right), \\ -2 \epsilon+(1 / \lambda)\left[1-e^{\lambda\left(x-\beta_{1}-\epsilon\right)}\right] & \text { in }\left(\beta_{1}+\epsilon, \beta_{2}-\epsilon\right) .\end{cases}
$$

Then $L f(x) \leq-b_{0}<0$ in $\left(\beta_{1}-\epsilon, \beta_{1}+\epsilon\right)$ and, for some positive number $\nu$,

$$
L f(x)=-b e^{k\left(x-\beta_{1}+\epsilon\right)}-1 / 2 k \sigma^{2} e^{k\left(x-\beta_{1}+\epsilon\right)}<-\nu
$$

in $\left(\alpha, \beta_{1}-\epsilon\right)$ provided $k$ is sufficiently large. Similarly $L f(x)<-\nu$ in $\left(\beta_{1}+\epsilon\right.$, $\left.\beta_{2}-\epsilon\right)$ if $\lambda$ is sufficiently large. Notice that $f^{\prime}(x)$ is continuous at $x=\beta_{1} \pm \epsilon$ but $f^{\prime \prime}(x)$ is discontinuous at the se points.

Set $f_{2}=f\left(\beta_{2}-\epsilon\right), \hat{f}_{2}=e^{\lambda\left(\beta_{2}-\beta_{1}-2 \epsilon\right)}$ and define

$$
f(x)= \begin{cases}-\hat{f}_{2}\left(x-\beta_{2}+\epsilon\right)+f_{2} & \text { in }\left(\beta_{2}-\epsilon, \beta_{2}+\epsilon\right), \\ -2 \epsilon \hat{f}_{2}+f_{2}+\left(\hat{f}_{2} / \mu\right)\left[1-e^{\mu\left(x-\beta_{2}-\epsilon\right)}\right] & \text { in }\left(\beta_{2}+\epsilon, \beta_{3}-\epsilon\right) .\end{cases}
$$

Then $f^{\prime}(x)$ is continuous at $x=\beta_{2} \pm \epsilon$, but $f^{\prime \prime}(x)$ is discontinuous at these points. It is easily seen that $L f(x)<-\nu<0$ in $\left(\beta_{2}-\epsilon, \beta_{2}+\epsilon\right)$ and, if $\mu$ is sufficiently large, also in $\left(\beta_{2}+\epsilon, \beta_{3}-\epsilon\right)$. We can proceed in this manner step by step to construct $f$ in the whole interval $(\alpha, \gamma)$.

We proceed to prove that

$$
E_{x}(\alpha, \gamma)<\infty \text { for any } x \in(\alpha, \gamma) \text {. }
$$

Let $\sigma_{n}, b_{n}$ be $C^{2}$ functions, $\sigma_{n}(x)>0$ for all $x, \sigma_{n}(x)=1, b_{n}(x)=0$ if $x<\alpha-1$, or if $x>y+1$, and as $n \rightarrow \infty, \sigma_{n}(x) \rightarrow \sigma(x), b_{n}(x) \rightarrow b(x)$ uniformly in $[a, \gamma]$. We shall first show that

$$
E \tau_{x}^{n}(\alpha, \gamma) \leq C<\infty
$$

where $C$ is a constant independent of $n$, and $\tau_{x}^{n}=\tau_{x}^{n}(a, \gamma)$ is the exit time from $(a, \gamma)$ of the stochastic process $\dot{\phi}_{n}(t)$ (with $\phi_{n}(0)=x$ ) corresponding to $\sigma_{n}, b_{n}$.

The function $f(x)$ constructed above for $\sigma, b$ is good also for $\sigma_{n}, b_{n}$ if $n$ is sufficiently large, that is,

$$
L_{n} f=(1 / 2) \sigma_{n}^{2} f^{\prime \prime}+b_{n} f^{\prime}<-\nu \quad \text { in }(\alpha, \gamma),
$$

except at a finite number of points $\gamma_{1}, \ldots, \gamma_{l}$ where $f^{\prime \prime}(x)$ has a jump; $\nu$ is a positive constant independent of $n$.

Let $\left\{g_{m}(x)\right\}$ be a sequence of continuous functions which approximate $f^{\prime \prime}(x)$ in the following manner: $g_{m}(x)=f^{\prime \prime}(x)$ if $\left|x-\gamma_{j}\right|>(1 / m)$ for all $1 \leq j \leq l$, and $g_{m}(x)$ connects $f^{\prime \prime}\left(\gamma_{j}-1 / m\right)$ to $f^{\prime \prime}\left(\gamma_{j}+1 / m\right)$ linearly. It is clear that the functions

$$
f_{m}(x)=f(\alpha)+f^{\prime}(\alpha) x+\int_{\alpha}^{x} \int_{\alpha}^{y} g_{m}(z) d z d y
$$


satisfy $f_{m}(x) \rightarrow f(x), f_{m}^{\prime}(x) \rightarrow f^{\prime}(x)$ uniformly in $(\alpha, \gamma)$, as $m \rightarrow \infty$, and $f_{m}^{\prime \prime}(x)=g_{m}(x)$.

Since $f_{m}$ is in $C^{2}$, we can apply Itô's formula and get

$$
E\left\{f_{m}\left(\sigma_{n}\left(\tau_{x}^{n} \wedge T\right)\right)\right\}-f_{m}(x)=E\left\{\int_{0}^{\tau_{x}^{n} \wedge T} L_{n} f_{m}\left(\phi_{n}(s)\right) d s\right\}
$$

for any $T>0$. Let

$$
E_{m}^{j}=\left\{(s, \omega) ; 0 \leq s \leq \tau_{n} \wedge T,\left|\phi_{n}(s)-\gamma_{j}\right|<1 / m\right\} .
$$

Let $d \mu$ be $d t \times d P$. Then

$$
\begin{aligned}
\mu\left(E_{m}^{j}\right) & \leq E\left\{\int_{0}^{T} X_{\left(\gamma_{j}-1 / m, \gamma_{j}+1 / m\right)}\left(\phi_{n}(s)\right) d s\right\} \\
& =\int_{0}^{T} d t \int_{\left|y-\gamma_{j}\right|<1 / m} p_{n}(t, x, y) d y \rightarrow 0 \text { if } m \rightarrow \infty
\end{aligned}
$$

where $p_{n}(t, x, y)$ is the transition density for the diffusion processes corresponding to $\sigma_{n}, b_{n}$; it exists by our assumptions on $\sigma_{n}, b_{n}$.

Since $L_{n} f_{m}\left(\phi_{n}(s)\right)$ is a bounded function of $(s, \omega)$ and since $L_{n} f_{m}\left(\phi_{n}(s)\right)=$ $L_{n} f\left(\phi_{n}(s)\right)$ for all $(s, \omega)$ not in $\bigcup_{j} E_{m}^{j}$, we can apply the Lebesgue bounded convergence theorem in passing to the limit $(m \rightarrow \infty)$ on the right-hand side of (3.19). Since the left-hand side is bounded by a constant $C^{\prime}$ independent of $m$, $n$, we get

$$
-C^{\prime} \leq E \int_{0}^{\tau^{n} \wedge T} L_{n} f\left(\phi_{n}(s)\right) d s
$$

the set of points $(s, \omega)$ at which $f\left(\phi_{n}(s)\right)=\gamma_{j}$ is of measure zero. Using (3.18) we conclude that $\nu E\left(\tau_{x}^{n} \wedge T\right) \leq C^{\prime}$, i.e., (3.17) holds. The same proof gives

$$
E\left\{\tau_{x}^{n}(\alpha-\eta, \gamma+\eta)\right\} \leq C
$$

if $\eta$ is sufficiently small.

Now, for each $T>0, \sup _{0 \leq s \leq T} E\left|\phi_{n}(s)-\phi(s)\right|^{2} \rightarrow 0$. Using this and the martingale inequality, we get for any $\mu>0$,

$$
\begin{aligned}
P\left\{\sup _{0 \leq t \leq T}\left|\phi_{n}(t)-\phi(t)\right|>2 \mu\right\} \leq & P\left\{\int_{0}^{T}\left|b_{n}\left(\phi_{n}(s)\right)-b(\phi(s))\right| d s>\mu\right\} \\
& +P\left\{\sup _{0 \leq t \leq T}\left|\int_{0}^{t}\left[\sigma_{n}\left(\phi_{n}(s)\right)-\phi(\phi(s))\right] d w\right|>\mu\right\} \\
& \rightarrow 0 \text { if } n \rightarrow \infty .
\end{aligned}
$$

Consequently,

$$
\tau_{x}(\alpha, \gamma) \wedge T \leq \frac{\lim }{n \rightarrow \infty} \tau_{x}^{n}(\alpha-\eta, \gamma+\eta) \wedge T
$$


Employing Fatou's lemma and (3.20), we find that $E\left\{\tau_{x}(\alpha, \gamma) \wedge T\right\} \leq C$. Taking $T / \infty$ the assertion (3.12) follows.

To prove (3.13), approximate $\sigma(x)$ uniformly in $\left(\beta_{0}, \beta_{k+1}\right)$ by Lipschitz continuous functions $\sigma_{n}(x)$ such that $\sigma_{n}\left(\beta_{1}\right)=0, \sigma_{n}(x)>0$ if $\beta_{1}<x<\gamma+\eta$, for some $0<\eta<\beta_{k+1}-\gamma$. From the first part of the lemma we have $E\left\{\tau_{x}^{n}\left(\beta_{1}, \gamma+\eta\right)\right\}<\infty$ where $\tau^{n}$ is the exit time corresponding to $\sigma_{n}, b$. Hence by Theorem 2 of $[4$, p. 150],

$$
P\left\{\phi_{n}\left(\tau_{x}^{n}\left(\beta_{1}, \gamma+\eta\right)\right)=\gamma+\eta\right\}=1
$$

for any $n$. Since $\tau_{x}\left(\beta_{1}, \gamma\right)$ is finite a.s., and since for any $T>0$

$$
P\left\{\sup _{0 \leq t \leq T}\left|\phi_{n}(t)-\phi(t)\right|>1 / 2 \eta\right\} \rightarrow 0 \text { if } n \rightarrow \infty,
$$

(3.13) follows.

Theorem 3.3. Assume that $\sigma(z), b(z)$ are periodic of period $2 \pi$, and that there is only a finite number of points $\beta_{1}<\beta_{2}<\cdots<\beta_{k}$ in $[0,2 \pi)$ where $\sigma(z)$ vanishes. Assume also that either (i) $b\left(\beta_{j}\right)>0$ for $1 \leq j \leq k$, or (ii) $b\left(\beta_{j}\right)<0$ for $1 \leq j \leq k$. Let

$$
T_{n}=\inf \left\{t ; \phi(t)-\phi_{0}=2 n \pi\right\} \quad(n= \pm 1, \pm 2, \ldots) .
$$

If (i) bolds then $0<E\left(T_{1}\right)<\infty$,

$$
P_{\phi_{0}}\left\{\lim _{t \rightarrow \infty} \frac{\phi(t)}{t}=\frac{2 \pi}{E\left(T_{1}\right)}\right\}=1
$$

and

$$
2 n \pi<\phi(t)<2(n+1) \pi \text { when } T_{n}<t<T_{n+1} .
$$

If (ii) bolds then $0<E\left(T_{-1}\right)<\infty$,

and

$$
P_{\phi_{0}}\left\{\lim _{t \rightarrow \infty} \frac{\phi(t)}{t}=-\frac{2 \pi}{E\left(T_{-1}\right)}\right\}=1
$$

$$
-2(n+1) \pi<\phi(t)<-2 n \pi \text { when } T_{-n}<t<T_{-n-1} .
$$

Proof. The assertions $E\left(T_{1}\right)<\infty$ and (3.22) (in case (i) holds) follow from Theorem 3.2. As in the proof of Theorem 3.1, the strong law of large numbers can be employed to deduce that $T_{n} / n \rightarrow E\left(T_{1}\right)$ a.s. if $n \rightarrow \infty$. Combining this with (3.22), the assertion (3.21) follows. The proof of (3.23), (3.24), in case (ii) holds, is similar.

4. One stochastic equation (continued). Consider the case where

$$
\sigma(\alpha)=\sigma(\beta)=0, \quad \sigma(x)>0 \quad \text { if } \alpha<x<\beta,
$$




$$
b(\alpha)>0, \quad b(\beta)<0 .
$$

Let $\alpha<\lambda<\mu<\beta$ and take $\phi_{0} \in(\lambda, \mu)$. Denote by $\tau^{1}$ the first time $\phi(t)$ exits $(\lambda, \mu)$ at $\mu$, and by $\tau^{2}$ the first time $>\tau^{1}$ that $\phi(t)$ hits $\lambda$. In general, denote by $\tau^{2 n+1}$ the first time $>\tau^{2 n}$ that $\phi(t)$ hits $\mu$, and by $\tau^{2 n+2}$ the first time $>\tau^{2 n+1}$ that $\phi(t)$ hits $\lambda$. By Theorem 3.2 and its analog in case $b\left(\beta_{i}\right)<0$ for $1 \leq i \leq k$, $\phi(t)$ remains in the interval $(\alpha, \beta)$ for all $t>0$, and the times $\tau^{m}$ have finite expectation. By the strong Markov property, the random variables $\rho_{3}=\tau^{3}-\tau^{2}$, $\rho_{5}=\tau^{5}-\tau^{4}, \ldots$ are independent and identically distributed. Hence, by the strong law of large numbers,

$$
\left(\rho_{3}+\rho_{5}+\cdots+\rho_{2 n+1}\right) / n \rightarrow E\left(\rho_{3}\right) .
$$

Similarly,

$$
\left(\rho_{2}+\rho_{4}+\cdots+\rho_{2 n}\right) / n \rightarrow E\left(\rho_{2}\right)
$$

We sum up:

Theorem 4.1. Let (4.1), (4.2) bold. Then for any $a<\lambda<\mu<\beta$, if $\phi_{0} \in(\lambda, \mu)$ then a.s. $\phi(\dot{t})$ remains in $(\alpha, \beta)$ for all $t>0$ and it oscillates from $\lambda$ to $\mu$; the number $N(t)$ of oscillations in $(0, t)$ tends to $\infty$ as $t \rightarrow \infty$, in accordance with (4.3), (4.4).

Consider next the case where (4.1) holds and

$$
b(\alpha)<0, \quad b(\beta)>0 .
$$

We shall prove that for any $x \in(\alpha, \beta)$ and for any small $\epsilon>0$,

$$
E\left\{\tau_{x}(\alpha-\epsilon, \beta+\epsilon)\right\}<\infty \text {. }
$$

The proof is similar to the proof of (3.12), once we construct a function $f(x)$ with continuous $f^{\prime}$ and piecewise continuous $f^{\prime \prime}$ such that

$$
L f(x) \leq-\nu \quad \text { in }(\alpha-\epsilon, \beta+\epsilon)
$$

where $\nu$ is some positive number. Take

$$
f(x)= \begin{cases}x-\alpha & \text { if } \alpha-\epsilon<x<\alpha+\epsilon, \\ x-\alpha-\lambda(x-\alpha-\epsilon)^{2 p} & \text { if } \alpha+\epsilon<x<\beta-\epsilon, \\ \beta-\alpha-\epsilon-\lambda(\beta-\alpha-2 \epsilon)^{p}\left[1-2 \lambda p(\beta-\alpha-2 \epsilon)^{2 p-1}\right](x-\beta+\epsilon) .\end{cases}
$$

One easily verifies that if $p$ and $\lambda$ are sufficiently large then (4.7) is satisfied. Suppose 


$$
\begin{aligned}
& \alpha_{l+1}<\alpha_{l}<\cdots<\alpha_{1}<\alpha_{0}<\beta_{0}<\beta_{1}<\cdots<\beta_{k}<\beta_{k+1}, \\
& \sigma\left(\alpha_{i}\right)=0 \quad(0 \leq i \leq l+1), \quad \sigma\left(\beta_{j}\right)=0 \quad(0 \leq j \leq k+1) \\
& \sigma(x)>0 \quad \text { if } x \in\left(\alpha_{l+1}, \beta_{k+1}\right) \text { and } x \neq \alpha_{i}, x \neq \beta_{j} .
\end{aligned}
$$

Suppose also that

$$
b\left(\alpha_{i}\right)<0 \quad(0 \leq i \leq l), \quad b\left(\beta_{j}\right)>0 \quad(0 \leq j \leq k) .
$$

Then, for any $\alpha \in\left(\alpha_{l+1}, \alpha_{l}\right), \beta \in\left(\beta_{k}, \beta_{k+1}\right)$ we can construct in $(\alpha, \beta)$ a function $f(x)$ which is piecewise $C^{2}$ and satisfies $L f \leq-\nu<0$. This leads to the following result.

Theorem 4.2. If (4.8), (4.9) bold then for any $\alpha \in\left(\alpha_{l+1}, \alpha_{l}\right), \beta \in\left(\beta_{k}, \beta_{k+1}\right)$, $x \in(\alpha, \beta), E\left\{\tau_{x}(\alpha, \beta)\right\}<\infty$.

Notice (by Theorem 3.2) that after $\phi(t)$ exits $\left(\alpha_{0}, \beta_{0}\right)$, it does not re-enter this interval in any future time.

Let the assumptions of Theorem 4.2 hold, and let $\bar{\alpha} \in\left(\alpha_{1}, \alpha_{0}\right], \bar{\beta} \in\left[\beta_{0}, \beta_{1}\right)$. We shall compute the probability that $\phi(t)$ exists $(\bar{\alpha}, \bar{\beta})$ at $\bar{\beta}$. Let $\gamma \in\left(\alpha_{0}, \beta_{0}\right)$,

$$
\Phi(x)=\exp \left\{-\int_{\gamma}^{x} \frac{2 b(u)}{\sigma^{2}(u)} d u\right\} .
$$

It is easily seen that $\Phi(x)$ is a smooth function in $[\bar{\alpha}, \bar{\beta}]$ and

$$
\pi(x)=\int_{\gamma}^{x} \Phi(z) d z
$$

is a solution of $L \psi=0$. Hence, by Theorem 4.2 and Itô's formula (cf. [4, p. 115])

$$
P\left\{\phi\left(\tau_{x}(\bar{\alpha}, \bar{\beta})\right)=\bar{\beta}\right\}=(\pi(x)-\pi(\bar{\alpha})) /(\pi(\bar{\beta})-\pi(\bar{\alpha})) .
$$

So far we have considered only the cases where at the points $\lambda$ where $\sigma(\lambda)=0$ the drift $b(\lambda) \neq 0$. We shall now consider the case where both $\sigma, b$ vanish at some points. The proof of Theorem 1.1 (with $r^{-\epsilon}$ replaced by $(x \pm \lambda)^{-\epsilon}$ ) shows that when $\sigma(\lambda)=b(\lambda)=0, \phi(t)$ cannot intersect $\phi=\lambda$ for any $t>0$.

Suppose (4.1) hold and let $\alpha<\gamma<\beta$. Introduce $\Phi(x), \pi(x)$ as in (4.10), (4.11). The process $\eta(t)=\pi(\phi(t))$ satisfies

$$
d \eta=\hat{\sigma}(\eta) d w
$$

where $\hat{\sigma}(y)=\Phi\left(\pi^{-1}(y)\right) \sigma\left(\pi^{-1}(y)\right), \pi^{-1}$ being the inverse function of $\pi$. Denote by $t^{*}$ the exit time of $\eta(t)$ from the interval $(\pi(\alpha), \pi(\beta))$. Then the solution $\phi(t)$ of (3.1) satisfies

$$
\pi(\phi(t))=\eta(t) \text { if } t<t^{*} \cdot
$$

In particular, $\tau_{x}(\alpha, \beta)=t^{*}$ (if $\left.\phi(0)=x\right)$. 
Theorem 4.3. Let $\phi(0)=x, \alpha<x<\beta$. (i) If $\pi(\alpha)=-\infty, \pi(\beta)=\infty$ then $\alpha<\phi(t)<\beta$ for all $t>0$ and

$$
\varliminf_{t \rightarrow \infty} \phi(t)=\operatorname{linf}_{t>0} \phi(t)=\alpha \quad \text { a.s., } \quad \varlimsup_{t \rightarrow \infty} \phi(t)=\sup _{t>0} \phi(t)=\beta \quad \text { a.s. }
$$

(ii) If $\pi(\alpha)>-\infty, \pi(\beta)=\infty$ and $b(\alpha)=0$, then $\alpha<\phi(t)<\beta$ for all $t>0$ and

$$
\lim _{t \rightarrow \infty} \phi(t)=\inf _{t>0} \phi(t)=\alpha \quad \text { a.s., } \quad \sup _{t>0} \phi(t)<\beta \quad \text { a.s. }
$$

(iii) If $\pi(\alpha)=-\infty, \pi(\beta)<\infty$ and $b(\beta)=0$, then $\alpha<\phi(t)<\beta$ for all $t>0$ and

$$
\lim _{t \rightarrow \infty} \phi(t)=\sup _{t>0} \phi(t)=\beta \quad \text { a.s., } \quad \inf _{t>0} \phi(t)>\alpha \text { a.s. }
$$

(iv) If $\pi(\alpha)>-\infty, \pi(\beta)<\infty, b(\alpha)=0$, and $b(\beta)=0$, then $\alpha<\beta$ for all $t>0$ and

$$
\begin{aligned}
& P\left\{\lim _{t \rightarrow \infty} \phi(t)=\alpha\right\}=P\left\{\inf _{t>0} \phi(t)=\alpha\right\}=\frac{\pi(\beta)-\pi(x)}{\pi(\beta)-\pi(\alpha)}, \\
& P\left\{\lim _{t \rightarrow \infty} \phi(t)=\beta\right\}=P\left\{\sup _{t>0} \phi(t)=\beta\right\}=\frac{\pi(x)-\pi(\alpha)}{\pi(\beta)-\pi(\alpha)} .
\end{aligned}
$$

Proof. (i) Lemma 1 of $[4$, p. 115] applied to (4.13) gives

$$
P\left\{\sup _{t>0} \eta(t)=\infty\right\}=P\left\{\varlimsup_{t \rightarrow \infty} \eta(t)=\infty\right\}=1 .
$$

Since, by (4.14), sup $\eta(t)=\infty$ if and only if $\sup \phi(t)=\beta$, the second part of (i) follows. The proof of the first part is similar.

(ii) Since $b(\alpha)=0, \phi(t)$ cannot cross $\phi=\alpha$ for any $t>0$. It also cannot cross $\phi=\beta$ (since $\eta(t)<\infty$ for all $t$ ). Thus $a<\phi(t)<\beta$ for all $t>0$. Since $\eta(t)>\pi(\alpha)$ for all $t$, the proof of Lemma 2 in [4, p. 116] can be modified, replacing $x=-\infty$ by $x=\pi(\alpha)$. This yields the assertions of (ii).

The proof of (iii) is similar to the proof of (ii), and the proof of (iv) is obtained by modifying the proof of Lemma 3 in [4, p. 117], replacing $+\infty,-\infty$ by $\pi(\beta)$ and $\pi(\alpha)$ respectively. Here the assumption $b(\alpha)=b(\beta)=0$ ensures that $\eta(t)$ remains in $(\pi(\alpha), \pi(\beta))$ for all $t$.

Note that if $b(\beta)<0$ then $\pi(\beta)=\infty$, and if $b(\alpha)>0$ then $\pi(\alpha)=-\infty$. Hence, the only cases that have not been covered so far (by Theorems 3.3, 4.14.3) are

$$
b(\alpha)=0, \quad b(\beta)>0,
$$

and

$$
b(\alpha)<0, \quad b(\beta)=0 .
$$

If (4.15) holds, $\phi(t)>\alpha$ for all $t$, but $\phi(t)$ may cross $\phi=\beta$ (as is evident from Theorem 3.3). 
Theorem 4.4. Let (4.1), (4.15) bold and let $x \in(\alpha, \beta)$. If $\pi(\alpha)=-\infty$ then $\tau_{x}(\alpha, \beta)<\infty$ a.s. and

$$
P\left\{\phi\left(\tau_{x}(\alpha, \beta)\right)=\beta\right\}=1 \text {. }
$$

If $\pi(\alpha)>-\infty$ then $\phi(t)>\alpha$ a.s. for all $t>0$ and

$$
\begin{gathered}
P\left\{\phi\left(\tau_{x}(\alpha, \beta)\right)=\beta\right\}=(\pi(x)-\pi(\alpha)) /(\pi(\beta)-\pi(\alpha)), \\
P\left\{\underline{\lim }_{t \rightarrow \infty} \phi(t)=\alpha\right\}=P\left\{\inf _{t>0} \phi(t)=\alpha\right\}=\frac{\pi(\beta)-\pi(x)}{\pi(\beta)-\pi(\alpha)} .
\end{gathered}
$$

A similar result holds in case (4.15) is replaced by (4.16).

Proof. By Theorem 3.2, Ev $\tau_{x}(\alpha+\epsilon, \beta)<\infty$ for any $\epsilon>0$. We therefore have the formulas

$$
\begin{aligned}
& P\left\{\phi\left(\tau_{x}(\alpha+\epsilon, \beta)\right)=\beta\right\}=(\pi(x)-\pi(\alpha+\epsilon)) /(\pi(\beta)-\pi(\alpha+\epsilon)), \\
& P\left\{\phi\left(\tau_{x}(\alpha+\epsilon, \beta)\right)=\alpha+\epsilon\right\}=(\pi(x)-\pi(\alpha+\epsilon)) /(\pi(\beta)-\pi(\alpha+\epsilon)) .
\end{aligned}
$$

Since $\phi(t)$ does not intersect $\phi=\alpha$ at any time, one can easily show that

$$
\left\{\phi\left(\tau_{x}(\alpha, \beta)\right)=\beta\right\}=\bigcup_{\epsilon: 0}\left\{\phi\left(\tau_{x}(\alpha+\epsilon, \beta)\right)=\beta\right\} .
$$

Using this in (4.20) with $\epsilon \rightarrow 0$, the assertions (4.17), (4.18) follow. The assertion (4.19) follows from (4.21).

5. Behavior of the angular motion. Theorems 3.1, 3.3, 4.1-4.4 can be immediately applied to the equation (2.7) with $\widetilde{\sigma}, \widetilde{b}$ given by (2.5), (2.6). Notice that $(\tilde{\sigma}(\phi))^{2}$ is a homogeneous polynomial of degree 4 in $(\cos \phi, \sin \phi)$. Consequently, $\widetilde{\sigma}(\phi)$ is periodic of period $\pi$, and it can have at most two zeros in the interval $[0, \pi)$. The function $\tilde{b}(\phi)$ is also periodic of period $\pi$.

The following possibilities may take place:

(i) $\widetilde{\sigma}(\phi)$ has two distinct zeros $\phi_{1}, \phi_{2}$ in the interval $[0, \pi)$.

(ii) $\tilde{\sigma}(\phi)$ has one zero in the interval $[0, \pi)$.

(iii) $\widetilde{\sigma}(\phi)$ does not vanish in the interval $[0, \pi)$.

If (iii) holds then Theorem 3.1 can be applied. If (i) or (ii) holds then

Theorems 3.3, 4.1-4.4 can be applied; the asymptotic behavior of $\phi(t)$ will depend only on the sign of $\tilde{b}(\phi)$ at the points $\phi_{i}$ where $\tilde{\sigma}\left(\phi_{i}\right)=0$. The following simple lemma is therefore of crucial importance.

Lemma 5.1. If for some $\phi, \tilde{\sigma}(\phi)=0$, then $\tilde{b}(\phi)=\left\langle B \lambda, \lambda^{\perp}\right\rangle$.

Proof. By formula (2.5),

$$
(\widetilde{\sigma}(\phi))^{2}=\sum_{r=1}^{n}\left(\sum_{i, k=1}^{2} \sigma_{i r}^{k} \lambda_{k} \lambda_{i}^{\perp}\right)^{2}=\sum_{r=1}^{n}\left(S_{1 r} \sin \phi-S_{2 r} \cos \phi\right)^{2}
$$


where $S_{i r}=\sigma_{i r}^{1} \cos \phi+\sigma_{i r}^{2} \sin \phi(i=1,2)$. Next, by $(2.6)$,

$$
\begin{aligned}
\tilde{b}(\phi)-\left\langle B \lambda, \lambda^{\perp}\right\rangle & =-\left\langle a(\lambda) \lambda, \lambda^{\perp}\right\rangle=-\sum_{r=1}^{n} \sum_{i, j, k, s=1}^{2} \sigma_{i r}^{k} \lambda_{k} \sigma_{j r}^{s} \lambda_{s} \lambda_{i} \lambda_{j}^{\perp} \\
& =-\sum_{r=1}^{n}\left(S_{1 r} \cos \phi+S_{2 r} \sin \phi\right)\left(-S_{1 r} \sin \phi+S_{2 r} \cos \phi\right) .
\end{aligned}
$$

Since the right-hand side vanishes whenever $\widetilde{\sigma}(\phi)=0$, the assertion of the lemma follows.

By Lemma 5.1, the sign of $\tilde{b}(\phi)$, at the points where $\widetilde{\sigma}(\phi)=0$, depends only on the deterministic system (0.1). This enables us to compare the behavior of the solutions of the stochastic system with the behavior of the solutions of the deterministic system. We shall give one consequence of this observation.

Theorem 5.2. Let (i) or (ii) bold. If for the deterministic system (0.1) $(l=2) \phi(t) \rightarrow \infty$ as $t \rightarrow \infty$, then the same is true for the stochastic system $(0.2)$, i.e., if $x(t)=(r(t) \cos \phi(t), r(t) \sin \phi(t))$, then a.s. $\phi(t) \rightarrow \infty$ as $t \rightarrow \infty$; in fact $[\phi(t) / t] \rightarrow 2 \pi / E\left(T_{1}\right)\left(0<E\left(T_{1}\right)<\infty\right)$. Similarly, if $\phi(t) \rightarrow-\infty$ as $t \rightarrow-\infty$ for the deterministic system, then a.s. $[\phi(t) / t] \rightarrow-2 \pi / E\left(T_{-1}\right)$ as $t \rightarrow \infty$ $\left(0<E\left(T_{-1}\right)<\infty\right)$.

Proof. In the deterministic case, $|\phi(t)| \rightarrow \infty$ as $t \rightarrow \infty$ if and only if the origin is a focal point (spiral or vortex). This is the case if and only if the eigenvalues of $B$ are nonreal, i.e., if and only if $\left\langle B \lambda, \lambda^{\perp}\right\rangle \neq 0$ for all $\lambda=$ $(\cos \phi, \sin \phi)$. Now use Lemma 5.1 and Theorem 3.3.

If the eigenvalues of $B$ are real (of the same sign for nodal points, and of different sign for saddle points) then $\left\langle B \lambda, \lambda^{\perp}\right\rangle$ does not have a fixed (positive or negative) sign. Nevertheless, the stochastic solutions may still spiral in accordance with Theorems $3.1,3.3$ if either (iii) holds and $\widetilde{\Lambda}$ (defined in (0.4)) is $\neq 0$, or if (ii) holds and $\left\langle B \lambda_{1}, \lambda_{1}^{\perp}\right\rangle \neq 0$, or if (i) holds and $\left\langle B \lambda_{i}, \lambda_{i}^{\perp}\right\rangle$ is positive for $i=1,2$ or negative for $i=1,2$; here $\lambda_{i}=\left(\cos \phi_{i}\right.$, sin $\left.\phi_{i}\right)$. In all other cases, the stochastic solution does not spiral, but Theorems 4.1-4.4, in conjunction with Lemma 5.1, can immediately be applied.

6. Application to the Cauchy problem. Consider the Cauchy problem

$$
\begin{array}{ll}
\partial u / \partial t=L u \quad \text { for } t>0, \\
u(0, x)=f(x) \quad \text { in } R^{l}(l=2)
\end{array}
$$

where $L u$ is defined by (1.2), (1.3) with $l=2$. Note that $L$ is a degenerate elliptic operator with unbounded coefficients. Suppose the second derivatives of $f(x)$ are continuous and 


$$
\left|D_{x}^{j} f(x)\right| \leq C\left(1+|x|^{b}\right) \quad(0 \leq j \leq 2 ; b>0)
$$

where $C, b$ are constants. Then there exists a unique solution of (6.1) satisfying in each strip $0 \leq t \leq T$,

$$
|u(t, x)| \leq M\left(1+|x|^{b}\right)
$$

where $M$ is a constant depending on $T$. Indeed, if $\xi_{x}(t)$ denotes the solution of (1.1) with $\xi_{x}(0)=x$ then, by standard arguments [4], the function

$$
u(t, x)=E\left\{f\left(\xi_{x}(t)\right)\right\}
$$

is a solution of (6.1). The uniqueness, even within the larger class of functions $u(t, x)$ satisfying a polynomial growth $|u(t, x)| \leq C\left(1+|x|^{k}\right)$ in each strip $0 \leq t \leq T$, where $C, K$ are some positive constants depending on $u$ and $T$, is proved in Problem 2 of [2, p. 56]. In the sequel, by a solution of the Cauchy problem $(6.1)$ we shall mean the function given by $(6.4)$ (even when $f$ is not continuous).

With $\widetilde{\sigma}, \tilde{b}$ defined in (2.5), (2.6), we make the following assumptions:

(A) If $\widetilde{\sigma}(\phi) \neq 0$ for all $\phi$ then $\tilde{\Lambda}$ (in $(0.4))$ is $\neq 0$; if $\widetilde{\sigma}(\phi)$ vanishes in $[0, \pi)$ and its zeros are $\phi_{1}, \phi_{2}$ (they may coincide) then either $\left\langle B \lambda_{i}, \lambda_{i}^{\perp}\right\rangle$ is positive for $i=1,2$ or $\left\langle B \lambda_{i}, \lambda_{i}^{\perp}\right\rangle$ is negative for $i=1,2$.

(B) $f(x)$ is independent of the radial variable, that is, there is a function $g(\phi)$ periodic of period $2 \pi$ such that $f(x)=g(\phi)$ if $x=(r \cos \phi, r \sin \phi)$.

Notice that if (A) holds then the stochastic solution $\phi(t)$ spirals and either $E\left(T_{1}\right)<\infty$ (if $\left.\phi(t) \rightarrow \infty\right)$ or $E\left(T_{-1}\right)<\infty($ if $\dot{\phi}(t) \rightarrow-\infty)$. We shall need the following lemma:

Lemma 6.1. If $(\mathrm{A})$ bolds with $E\left(T_{1}\right)<\infty$ then $T_{1}$ is not concentrated on any lattice of the form $\{n \alpha ; n=1,2, \ldots\}$, a real.

Proof. The assertion of the lemma is equivalent to the assertion that the equation $1=E\left\{e^{i y T^{1}}\right\}$ ( $y$ real) has a unique solution $y=0$. In proving the lemma we shall use the fact that $\widetilde{\sigma}(\phi)$ does not vanish identically, i.e., there is a point $\phi_{0} \in(0,2 \pi)$ and a $\delta>0$ such that $\widetilde{\sigma}(\phi)>0$ if $\left|\phi-\phi_{0}\right| \leq \delta$. We can take $\delta$ such that $0<\phi_{0}-\delta, \phi_{0}+\delta<2 \pi$. Let $I_{1}=\left[0, \phi_{0}-\delta\right), I_{2}=\left[\phi_{0}-\delta, \phi_{0}+\delta\right), I_{3}=$ $\left[\phi_{0}+\delta, 2 \pi\right)$, and let $T_{1}=U_{1}+U_{2}+U_{3}$ where $U_{1}=\inf \left\{t>0 ; \phi(t)=\phi_{0}-\delta\right\}$, $U_{2}=\inf \left\{t>0 ; \phi(t)=\phi_{0}+\delta\right\}, U_{3}=\inf \{t>0 ; \phi(t)=2 \pi\}$. By the strong Markov property,

$$
E_{0}\left(e^{i y T} 1\right)=E_{0}\left(e^{i y U_{1}}\right) E_{\phi_{0}-\delta}\left(e^{i y U_{2}}\right) E_{\phi_{0}+\delta}\left(e^{i y U_{3}}\right) .
$$

Since $\left|e^{i y U_{j}}\right| \leq 1$, each expectation on the right-hand side is $\leq 1$. Thus, in order to complete the proof of the lemma it suffices to show that $\left|E_{\phi_{0}-\delta}\left(e^{i y U_{2}}\right)\right|<1$ if $y \neq 0$. This is equivalent to showing that, when $\phi(0)=\phi_{0}-\delta, U_{2}$ is not concentrated on a lattice of the form $\{\alpha+n \beta ; n=1,2, \cdots\} ; \alpha, \beta$ real. For this it 
suffices to show that $P_{\phi_{0}-\delta}\left(U_{2}<t\right)>0$ for any $t>0$. But the function $w(t, x)$ $=P_{x}\left(U_{2}<t\right)$ for $t>0,-\delta-\epsilon \leq x-\phi_{0} \leq \delta(\epsilon>0$ and sufficiently small $)$ satisfies

$$
\partial w / \partial t=1 / 2(\widetilde{\sigma}(x))^{2} \partial^{2} w / \partial x^{2}+\tilde{b}(x) \partial w / \partial x,
$$

and $w(0, x)=0$ if $-\delta-\epsilon \leq x-\phi_{0} \leq \delta, w\left(t, \phi_{0}+\delta\right)=1$ if $t>0$. Since $\tilde{\sigma}(x)>0$ if $-\delta-\epsilon \leq x-\phi_{0} \leq \delta$ and $\epsilon$ is sufficiently small, the strong maximum principle [2] can be applied to conclude that $w(t, x)>0$ if $t>0,-\delta-\epsilon \leq x-\phi_{0} \leq \delta$. Hence $P_{\phi_{0-\delta}}\left(U_{2}<t\right)>0$.

Theorem 6.2. Let (A), (B) bold. Then the solution $u(t, x)$ of the Caucby problem (6.1) satisfies $\lim _{t \rightarrow \infty} u(t, x)$ exists for any $x \in R^{2}, x \neq 0$.

Proof. It suffices to prove the theorem in case $\phi(t) \rightarrow \infty$ as $t \rightarrow \infty$. In this case $E\left(T_{1}\right)<\infty$. Consider the function $u(t)=E\{g(\phi(t))\}$ where $\phi(t)$ is the solution of (2.7) with $\phi_{0}=x$. What we have to prove is that

$$
\lim _{t \rightarrow \infty} u(t) \text { exists. }
$$

Note that $u(t)$ is a bounded function.

We introduce the Laplace transform $V(s)=\int_{0}^{\infty} e^{-s t} u(t) d t, s=\lambda+i \mu, \lambda>0$. Then

$$
\begin{aligned}
V(s) & =E\left\{\int_{0}^{\infty} e^{-s t} g(\phi(t)) d t\right\}=E\left\{\sum_{n=1}^{\infty} \int_{T_{n-1}}^{T_{n}} e^{-s t} g(\phi(t)) d t\right\} \\
& =\sum_{n=1}^{\infty} E\left\{e^{-s T_{n-1}} \int_{0}^{T_{1} \circ \theta_{n-1}} e^{-s t} g\left(\phi(t) \circ \theta_{T_{n-1}}\right) d t\right\}
\end{aligned}
$$

where $\theta_{T}$ is the translation operator. Using the strong Markov property and the representation $T_{n-1}=T_{1}+\left(T_{2}-T_{1}\right)+\cdots+\left(T_{n-1}-T_{n-2}\right)$ of $T_{n-1}$ as a sum of independent and identically distributed random variables, we get

$$
V(s)=\sum_{n=0}^{\infty}\left[E\left(e^{-s T_{1}}\right)\right]^{n} E\left\{\int_{0}^{T}{ }^{1} e^{-s t} g(\phi(t)) d t\right\}
$$

$$
=E \int_{0}^{T} 1 e^{-s t} g(\phi(t)) d t /\left(1-E\left(e^{-s T_{1}}\right)\right) .
$$

If $\lambda \geq 0, s \neq 0$, then

$$
\operatorname{Re}\left\{1-E\left(e^{-s T_{1}}\right)\right\}=\int_{0}^{\infty}\left(1-e^{-\lambda t} \cos \mu t\right) P\left(T_{1} \in d t\right)>0
$$

by Lemma 6.1 . Hence

$$
1-E\left(e^{-s T_{1}}\right) \neq 0 \text { if } \lambda \geq 0, s \neq 0 .
$$

We can write 
(6.8) $E\left\{\int_{0}^{T} 1 e^{-s t} g(\phi(t)) d t\right\}=\int_{0}^{\infty} e^{-s t} E\left\{g(\phi(t)) \chi_{\left(t \leq T_{1}\right)}\right\} d t=\int_{0}^{\infty} e^{-s t} k(t) d t$

where

$$
k(t)=E\left\{g(\phi(t)) \chi_{\left(t \leq T_{1}\right)}\right\} \text { belongs to } L^{1}(0, \infty)
$$

since $E\left(T_{1}\right)<\infty$.

Consider the function

(6.10) $\psi(s)=\left(1-E\left(e^{-s T}\right)\right) / s=\int_{0}^{\infty}\left(\left(1-e^{-s t}\right) / s\right) P\left(T_{1} \in d t\right)=\int_{0}^{\infty} e^{-s t} P\left(T_{1} \geq t\right) d t$.

Since $E\left(T_{1}\right)<\infty$ the integrand

$$
b(t)=P\left(T_{1} \geq t\right) \text { belongs to } L^{1}(0, \infty) .
$$

We now introduce the constant $A=E\left\{\int_{0}^{T}{ }^{1} g(\phi(t)) d t\right\}$ and consider the functions $\tilde{u}(t)=u(t)-A, \tilde{g}(t)=g(t)-A$. Then $\tilde{u}(t)=E\{\tilde{g}(\phi(t))\}$ and, by (6.6), (6.8), (6.10),

$$
\widetilde{V}(s) \equiv \int_{0}^{\infty} e^{-s t} \widetilde{u}(t) d t=\frac{1}{s} \frac{\int_{0}^{\infty} e^{-s t} E\left\{\tilde{g}(\phi(t))_{\left(t \leq T_{1}\right)}\right\} d t}{\int_{0}^{\infty} e^{-s t} P\left(T_{1} \geq t\right) d t} \equiv \frac{1}{s} \frac{N(s)}{D(s)}
$$

where $N(s)$ stands for the numerator and $D(s)$ stands for the denominator. We would like to apply the tauberian theorem of Landau-Ikehara [8, p. 130] which states:

If $\tilde{u}(t)$ is bounded below, if $\tilde{u}(t) e^{-\lambda t} \in L^{1}(0, \infty)$ for any $\lambda>0$, if $\tilde{V}(i \mu) \equiv$ $\lim _{\lambda \rightarrow 0} \tilde{V}(\lambda+i \mu)$ exists for almost all $\mu$ and is locally integrable, and if

$$
\lim _{\lambda \rightarrow 0} \int_{-M}^{M}|\widetilde{V}(\lambda+i \mu)-\widetilde{V}(i \mu)| d \mu \rightarrow 0
$$

for any $M>0$, then, for any $\delta>0$,

$$
\int_{x-\delta}^{x+\delta} \tilde{u}(t) d t \rightarrow 0 \text { if } x \rightarrow \infty
$$

In our case, $\tilde{u}(t)$ is uniformly bounded and from (6.6)-(6.11) it is clear that $\tilde{V}(i \mu) \equiv \tilde{V}(i \mu+0)$ exists and is bounded for $\mu$ in any closed bounded interval $I$ that does not intersect the origin, and

$$
\int_{I}|\widetilde{V}(\lambda+i \mu)-\widetilde{V}(i \mu)| d \mu \rightarrow 0 \text { if } \mu \rightarrow 0 .
$$

To study the behavior of $\tilde{V}(s)$ near $s=0$, write

$$
\frac{N(s)}{s}=\int_{0}^{\infty} \frac{e^{-\lambda t-i \mu t}-1}{\lambda+\mu i} E\left\{g(\phi(t))_{\left(t \leq T_{1}\right)}\right\} d t \text {. }
$$


The fraction in the integrand is bounded by $C^{\prime} t$, where $C^{\prime}$ is a constant, and

$$
\int_{0}^{\infty} t\left|E\left\{g(\phi(t)) \chi_{\left(t \leq T_{1}\right)}\right\}\right| d t \leq C \int_{0}^{\infty} t E\left\{\chi_{\left(t \leq T_{1}\right)}\right\} d t \leq C E\left\{T_{1}^{2}\right\}<\infty .
$$

(In fact, since $E_{x}\left(T_{1}\right) \leq c<\infty$ for all $x$, where $c$ is a constant, the strong Markov property implies that $E\left\{e^{\gamma T_{1}}\right\}<\infty$ for some $\gamma>0$.) It follows that

$$
\frac{N(s)}{s} \rightarrow-\int_{0}^{\infty} t E\left\{g(\phi(t)) \chi_{\left(t \leq T_{1}\right)}\right\} d t \text { if } s \rightarrow 0,
$$

and the integral is finite. Recalling (6.12) we easily conclude that (6.15) holds also for bounded intervals $I$ which contain the origin. Thus the assumption (6.13) in the above cited tauberian theorem is satisfied. The assertion (6.14) is therefore valid.

Now, as easily seen, for any $t, t^{\prime}$ in $[0, \infty)$,

$$
E\left|\phi(t)-\phi\left(t^{\prime}\right)\right|^{2} \leq C\left|t-t^{\prime}\right| \quad(C \text { constant }) .
$$

Since $g(\phi)$ is Lipschitz continuous, we get

$$
\left|u(t)-u\left(t^{\prime}\right)\right| \leq C\left|t-t^{\prime}\right|^{1 / 2}
$$

with another constant $C$. Hence,

$$
\left|2 \delta \widetilde{u}(t)-\int_{t-\delta}^{t+\delta} \widetilde{u}\left(t^{\prime}\right) d t^{\prime}\right| \leq 2 C \delta^{3 / 2}
$$

Using (6.14) we conclude that $\overline{\lim }_{t \rightarrow \infty}|\widetilde{u}(t)| \leq 2 C \delta^{1 / 2}$, i.e., $\tilde{u}(t) \rightarrow 0$ if $t \rightarrow \infty$. This completes the proof of (6.5).

As a by-product of the proof we obtain

(6.16) $\lim _{t \rightarrow \infty} u(t, x)=E\left\{\int_{0}^{T_{1}} g(\phi(t)) d t\right\}$, where $x=(\cos \phi(0), \sin \phi(0))$.

Consider now the case of a general initial function $f(x)$ and suppose that

$$
f(r \cos \phi, r \sin \phi) \rightarrow g(\phi) \text { uniformly as } r \rightarrow \infty \text {. }
$$

Suppose also that

$$
Q(\lambda)>0 \text { for all } \lambda=(\cos \phi, \sin \phi)
$$

where $Q(\lambda)$ is as in (1.4). Then we can state

Corollary 6.3. If (A), (6.17) and (6.18) bold, then the assertion of Theorem 6.2 is valid.

Indeed, by Theorem 1.2, $r(t) \rightarrow \infty$. This and (6.17) imply that

$$
u(t, x)-E\{g(\phi(t))\} \rightarrow 0 \text { if } t \rightarrow \infty \text {. }
$$

Now use Theorem 6.2. 
We shall give another application to the Cauchy problem. Let

$$
\widetilde{\sigma}\left(\phi_{1}\right)=\widetilde{\sigma}\left(\phi_{2}\right)=0 \quad \text { where } 0 \leq \phi_{1}<\phi_{2}<\pi
$$

and suppose that

$$
\left\langle B \lambda_{1}, \lambda_{1}^{\perp}\right\rangle>0, \quad\left\langle B \lambda_{2}, \lambda_{2}^{\perp}\right\rangle<0
$$

where $\lambda_{i}=\left(\cos \phi_{i}, \sin \phi_{i}\right), \lambda_{i}^{\perp}=\left(-\sin \phi_{i}, \cos \phi_{i}\right)$. Denote by $S$ the set $\left\{(r, \phi) ; r>0, \phi_{1}<\phi<\phi_{2}\right.$ or $\left.\phi_{1}+\pi<\phi<\phi_{2}+\pi\right\}$. $S$ consists of two sectors.

From Theorem 4.1 we deduce that if $x \in S$ then $\xi_{x}(t) \in S$ for all $t>0$. Using (6.4) we conclude

Theorem 6.4. If (6.19), (6.20) bold then for any $x \in S$ the solution $u$ of (6.1) at the points $(t, x), t>0$, depends only on the initial data f restricted to $S$.

Thus the domain of dependence of the set $\{(t, x) \in[0, \infty) \times S\}$ is $S$.

Further results of the same nature can be obtained by employing Theorems 4.2-4.4.

\section{REFERENCES}

1. M. I. Frĕ́dlin, The exterior Dirichlet problem for the class of bounded functions, Teor. Verojatnost. i Primenen. 11 (1966), 463-471 = Theor. Probability Appl. 11 (1966), 407-414. MR 34 \#1694.

2. A. Friedman, Partial differential equations of parabolic type, Prentice-Hall, Englewood Cliffs, N. J., 1964. MR 31 \#6062.

3. - Limit behavior of solutions of stochastic differential equations, Trans. Amer. Math. Soc. 170.(1972), 359-384.

4. I. I. Gihman and A. V. Skorohod, Stochastic differential equations, "Naukova Dumka", Kiev, 1968. (Russian) MR $41 \# 7777$.

5. R. Z. Has'minskir, Necessary and sufficient conditions for the asymptotic stability of linear stochastic systems, Teor. Verojatnost. i Primenen. 12 (1967), 167-172 = Theor. Probability Appl. 12 (1967), 144-147. MR 35 \#2345.

6. F. Kozin and S. Prodromou, Necessary and sufficient conditions for almost sure sample stability of linear Ito equations, SIAM J. Appl. Math. 21 (1971), 413-424.

7. H. P. McKean, Jr., Stochastic integrals, Probability and Math. Statist., no. 5, Academic Press, New York, 1969. MR 40 \#947.

8. H. R. Pitt, Tauberian theorems, Tata Institute of Fundamental Research Monographs on Math. and Physics, no. 2, Oxford Univ. Press, London, 1958. MR 21 \#5109.

DEPARTMENT OF MATHEMATICS, NORTHWESTERN UNIVERSITY, EVANSTON, ILLINOIS 60201 